\title{
Design and Implementation of Agricultural Machinery Management System Based on $C / S$ Mode
}

\author{
Muyang Li1)
}

\begin{abstract}
With the advancement of agricultural machinery information management process, this paper proposes a design and implementation of agricultural machinery management system based on $\mathrm{C} / \mathrm{S}$ structure. Using the $\mathrm{C} / \mathrm{S}$ structure can effectively reduce the amount of data transmitted on the network, and the user has higher efficiency. The implemented agricultural machinery management system utilizes a database to manage daily agricultural machinery data, and the log can accurately query each business for verification. This not only reduces the probability of errors, but also greatly improves the efficiency of agricultural machinery market management.
\end{abstract}

Keywords: Agricultural, Machinery, Information, Management, C/S Structure, Machinery, Data

\section{Introduction}

At the current stage, the degree of agricultural mechanization in China continues to increase, which brings about the continuous prosperity of the agricultural machinery market, precisely because there are so many agricultural machinery products demand[1]. In the daily management of the agricultural machinery market, how to manage various agricultural machinery products, various warehouses, and the distribution of management personnel has become an urgent problem to be solved at this stage. In recent years, during the sales of agricultural machinery products, the management level of the agricultural machinery market has significantly improved, and a lot of experience has been accumulated[2]. However, the current methods of publicity, sales and management of various agricultural machinery products are still traditional. There are many deficiencies in this method in terms of information collection, processing, and release, such as low efficiency, small amount of information contained, and poor feedback functions; the use of information is inefficient, the timeliness and system of management information are poor, Disadvantages such as insufficient decision-making basis[3]. Therefore, it is necessary to realize the information of agricultural machinery product 
management.

In order to deal with the informatization of agricultural machinery management, this paper designs and implements an agricultural machinery management system based on C/S structure. The C/S structure is easy and fast to implement. The system's management interface and database are built using Visual Studio 2012 and SQL-Server 2008. Through this platform, managers of the agricultural machinery market can quickly and easily complete each business, and can obtain various information such as inventory and sales status through statistical data in order to carry out the next sales strategy.

\section{Analysis of Main Functions of Agricultural Machinery Management System}

Information technology is an important development opportunity facing various industries in the society at present. The same is true for agricultural machinery sales and management industries. The use of information technology can enhance management efficiency, reduce management costs, and optimize enterprise sales strategies through decision support. In the informatization project, the enterprise continuously strengthens its core competitiveness, and can also continuously expand the development space of the enterprise[4]. In the daily management process of agricultural machinery product sales enterprises, the information-based agricultural machinery management system can complete the collection, arrangement and standardization of various information, and automatically complete various tasks required for management. Before developing the agricultural machinery management system, the first problem to be solved was system analysis. It can also be said that system analysis is the basis of development[5].

The agricultural machinery management system is generally applied to the daily agricultural machinery procurement and sales in the agricultural machinery market, which has higher requirements for the speed and processing capacity of data transactions and data security. Therefore, this system uses a C/S structure, that is, a client/server structure. Compared with the $\mathrm{B} / \mathrm{S}$ structure, the $\mathrm{C} / \mathrm{S}$ structure is better at data manipulation, transaction processing capabilities, and security and integrity constraints. Among them, all data of the server-side storage system and the relationship between the data, and the client builds a bridge between the system and users. Users operate the client to process server-side data. In order to complete various services, this method can effectively reduce network traffic and server computing. All business logic of the system is implemented using stored procedures, which are deployed on the database server and can be called by client applications in the foreground. The server side of the agricultural machinery management system is built using SQL-Server 2008, and stores all 
data tables of the system, including user tables, agricultural machinery product tables, warehouse tables, order tables, and log tables.

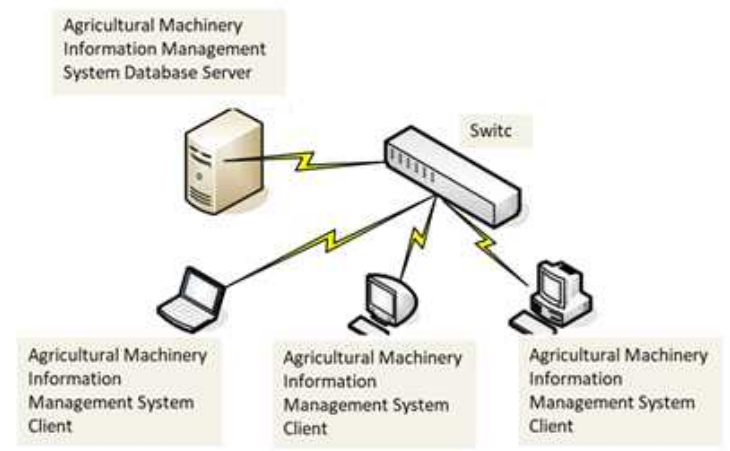

[Fig. 1] Schematic Diagram of C/S Structure of Agricultural Machinery Management System

As shown in [Fig. 1], all data of the server storage system and the relationship between the data are stored on the server-side of the agricultural management system, and the client builds a bridge between the system and the user through the interface application. Users operate the client to process server-side data. In order to complete various services, this method can effectively reduce network traffic and server computing. All business logic of the system is implemented using stored procedures, which are deployed on the database server and can be called by client applications in the foreground.

\section{Overall System Design}

The client program is written in Visual Studio 2012. The overall system structure is shown in [Fig. 2].

According to the functional requirements, the agricultural machinery management system is divided into five parts: system management module, procurement management module, inventory management, sales management and statistical decision-making module. The five main workflows of the agricultural machinery management system are mainly divided into the following aspects. 


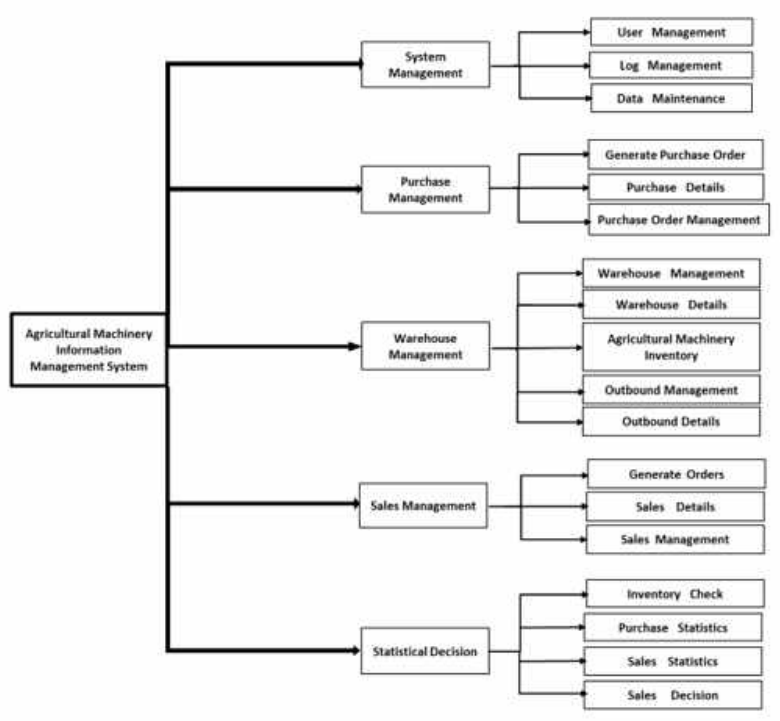

[Fig. 2] Agricultural Machinery Management System Structure

According to the functional requirements, the agricultural machinery management system is divided into five parts: system management module, procurement management module, inventory management, sales management and statistical decision-making module. The five main workflows of the agricultural machinery management system are mainly divided into the following aspects.

\subsection{System Management}

Only the system administrator can operate the system management part. This section includes user management, log management, and data maintenance. Users are divided into administrator users and ordinary users. Administrator users have all the functions of the operating system. Administrator users can assign permissions to ordinary users, and can separately assign permissions for purchasing management, inventory management, sales management, and statistical decision making. In the daily management of the agricultural machinery market, in order to facilitate statistical data and prevent business errors, every business operation should be backed up by logs. Key operations including user changes, warehouse operations, warehouse operations, warehouse settings, statistical data, price adjustments, etc., are stored in corresponding operation logs. Log management is able to view 
these logs and mark and comment on the corresponding logs for statistical queries in the future. The system maintenance module gives administrator users super authority to modify and delete all data for special and abnormal data processing. However, such super privilege operations are recorded in the $\log$ for the record.

\subsection{Purchasing Management}

Purchase management includes the generation of purchase orders, purchase details, and purchase order management components. The procurement process of agricultural machinery products is carried out with purchase orders as a unit. After the manager proposes purchase requirements, the purchaser needs to submit and generate a purchase order. The purchase order includes purchase order serial number, dealer number, purchase time, purchase staff and other information. Then fill in the purchase details of the various types of agricultural machinery products purchased, the sales price and quantity. In the purchase order management, you can modify and calculate the generated purchase order.

\subsection{Inventory Management}

Inventory management includes five parts: inventory management, inventory details, inventory inventory, inventory management, and inventory details. After the procurement of a batch of agricultural machinery products is completed, the batch of agricultural machinery products needs to be stored in a warehouse. Storage management includes purchase order number, dealer order number, operator, storage time and other information. Specific agricultural machinery product information is reflected in the storage details. In the daily operation of the warehouse, the warehouse needs to be checked at regular intervals to check the number of models of agricultural machinery products, as well as damage. The agricultural machinery products in the warehouse need to be counted. The content of inventory is reflected in the agricultural machinery inventory module. This includes information on inventory personnel, inventory time, and inventory results. During the sales of agricultural machinery products, the agricultural machinery products will be shipped out after the order is generated. The outbound management module generates the outbound order containing the outbound order number, the outbound time, and the operator. Product number, quantity, etc.

\subsection{Sales Management}


In the agricultural machinery management system, sales management is an important part. The sales management includes order generation, order details, and order management. In the sales process of agricultural machinery products, orders are first generated according to customer needs. The order includes information such as order number, order generation time, salesperson, order type, and so on. After the customer selects the agricultural machinery product to be purchased, the salesperson generates an order in the agricultural machinery management system, the system automatically generates the order number and the order generation time, and then manually fills in the order type, salesperson information, etc. In the order details, enter the information about the agricultural machine product and model that the customer needs to purchase, the order quantity, customer name, contact phone number, email address, and delivery address.

\subsection{Statistical Decisions}

The statistical decision module includes four functions: purchase statistics, inventory statistics, sales statistics, and sales decisions. Among them, the purchasing statistics can be based on time, operators, dealers and other keywords to query and statistics on the purchase order, so that management personnel can grasp various types of purchasing information. Inventory statistics refers to querying the current inventory and querying historical inbound and outbound records. Sales statistics can query historical orders and sales records, and can count the total turnover in the time period. By combining with purchasing statistics, you can also query the net profit in the time period. In the sales statistics function, it can also query the existing customer information and the customer's agricultural machinery product purchase situation. In the agricultural machinery management system based on data mining, the most critical function is the sales decision module. In the sales decision module, the decision maker finds the data association through the data mining algorithm based on historical sales data and customer sales records. The customer group is classified, and the final result is returned to the sales strategy reference of various customers, so that decision-makers can better choose the sales strategy, thereby improving the efficiency of agricultural machinery sales.

\section{Database Design}

The design of database tables is a key part in agricultural machinery management systems. These include user tables, agricultural product tables, warehouse tables, order tables, and log tables. 


\subsection{User Table}

In [Table 1], administrator of system can register the UserID, UserName and UserKey. The attribute UserPower is an authorization number.

[Table 1] User Table

\begin{tabular}{ccc}
\hline Attribute & Type & \\
\hline UserID & Varchar(10) & \\
UserName & Varchar(10) Key \\
UserKey & Varchar(10) & \\
UserPower & Varchar(10) & \\
Remark & Nvarchar(50) & \\
\hline
\end{tabular}

\subsection{Agricultural Machinery Product Table}

[Table 2] shows the attributes of the agricultural machinery. The machinery ID, machinery name, machinery type, version and agency should be given in system.

[Table 2] Agricultural Machinery Product

\begin{tabular}{ccc}
\hline Attribute & Type & - \\
\hline MachineryID & Varchar(10) & \\
MachineryName & Varchar(30) key \\
MachineryType & Varchar(10) & \\
Version & Varchar(10) & \\
Agency & Varchar(30) & \\
Remark & Nvarchar(100) & \\
\hline
\end{tabular}

\subsection{Warehouse Table}

Store room ID, StoreroomType, Machinery ID, Sale Price, number, storekeeper are given in Table 3. The primary key is Machinery ID.

[Table 3] Warehouse

\begin{tabular}{ccc}
\hline Attribute & Type & - \\
\hline StoreroomID & Varchar(10) & Primary Key \\
StoreroomType & Varchar(10) & \\
MachineryID & Varchar(10) & \\
SalePrice & Decimal &
\end{tabular}




\begin{tabular}{cc} 
Number & Int \\
Storekeeper & Varchar(10) \\
Remark & Nvarchar(50) \\
\hline
\end{tabular}

\subsection{Order Form}

Sales information is important thing for the system. In Table 4 - order form, Sale ID, Machinery ID, Sale number, sale date, transactor were given.

[Table 4] Order Table

\begin{tabular}{ccc}
\hline Attribute & Type & - \\
\hline SaleID & Varchar(10) & Primary key +foreign key \\
MachineryID & Varchar(10) & \\
SaleNumber & Int & \\
SaleDate & DateTime & Primary key \\
Transactor & Varchar(10) & \\
Remark & Nvarchar(50) & \\
\hline
\end{tabular}

\subsection{System Testing and Results}

\subsubsection{Testing Environment}

(1) Development platform: macOS High Sierra (10.13.6 (17G4015))

(2) Host: MacBook Air (11-inch, Early 2015)

(3) Processor: $1.6 \mathrm{GHz}$ Intel Core i5

(4) Memory: 4 GB $1600 \mathrm{MHz}$ DDR3

(5) Graphics card: Intel HD Graphics 60001536 MB 128 GB solid-state "PCI-Express" drive

(6) Development tools: eclipse (Eclipse IDE for Enterprise Java Developers.Version: 2018-12 (4.10.0) Build id: 20181214-0600) vistual studio code (version 1.30 .1 (1.30.1))

(7) Database: mysql for mac (8.0.13)

(8) Visual database tools: mysql workbench (8.0.13), Apache Tomcat / 7.0.92

\subsubsection{Function Testing}

In function testing, 3 staff in agricultural market were invited to finish the testing. Each staff did the testing (System Management, Purchase, Warehouse, Sales, and Statistical Decisions) with the frequency 3. At last, the test conclusion is "Proposed system is useful and efficiency" 


\begin{tabular}{cc}
\hline Testing & Content \\
\hline Test title & Function Tesing \\
Testing purposes & System Management, Purchase, Warehouse, Sales, Statistical Decisions \\
Testing frequency & 3 \\
expected outcome & Successful \\
Testing process & Each process in system structure \\
Test Results & Successful \\
Test conclusion & Proposed system is useful and efficiency \\
\hline
\end{tabular}

\subsubsection{Statistical Decisions Results}

In the statistical decisions process, data mining classification process is used. The monthly sales volume of agricultural products, the total monthly purchase amount, and the monthly gross profit margin are used as evaluation indicators. Classification is obtained through the weight of each indicator in the algorithm. Table shows part of the result data generated by the statistical decisions in agricultural machinery management system. It can be seen that the system could classify the customers according to their purchasing power levels. There are three grades: High, Medium, and low.

[Table 6] Customer Information Results Using Data Mining

\begin{tabular}{crlc}
\hline User No. & User name & User type & User Grade \\
\hline 230103100108 & Longfeng Agricultural Machinery Store & Company & High \\
230102700087 & Yuejin Agricultural Machinery Store & Company & High \\
230102000064 & Shengli Agricultural Machinery Distribution Office & Company & High \\
230101500129 & Tiansheng Farm Machinery Store & Company & High \\
230100400023 & Huabin Agricultural Machinery Store & Company & High \\
230100900002 & Xinsheng Agricultural Machinery Store & Company & High \\
230402300102 & Fuyuan Agricultural Machinery Distribution Office & Company & High \\
230103000003 & Vocational College of Agricultural Engineering & Company & High \\
230102300114 & Wangjia Agricultural Machinery Station & Company & Medium \\
230102700114 & Zhang Maocai & Personal & Medium \\
230101000129 & Zhao Futian & Personal & Medium \\
230101100287 & Sun Shengli & Personal & Medium \\
230100800367 & Zhang Jianhua & Personal & Medium
\end{tabular}




\begin{tabular}{lccc}
230100400723 & Li Xueyong & Personal & Low \\
230100700078 & Ge Yongguang & Personal & Low \\
230100200023 & Dai Gang & Personal & Low \\
230100800012 & Wang Jun & Personal & Low \\
230101000192 & Shi Lei & Personal & Low \\
230100800138 & Shan Degang & Personal & Low \\
\hline
\end{tabular}

\section{Conclusion}

This article details the design and implementation process of the agricultural machinery management system. The system provides a convenient and fast software platform for the daily management of the agricultural machinery market and improves the management efficiency. At the same time, the overall functional design of the agricultural machinery management system with $\mathrm{C} / \mathrm{S}$ architecture is given. The functional modules include system management, procurement management, inventory management, sales management, and statistical decision-making. The function of each function module is introduced.

\section{References}

[1] Li Yi, Chen Hongxing and Chen Peng, Status and prospect of agricultural machinery management informatization, Research on Agricultural Mechanization, (2004), Vol.5, pp.56-58.

[2] Meng Qingshan, A Preliminary Study on the Application Prospects of Farm Agricultural Machinery Information Management System, Proceedings of the Branch of the 2010 International Agricultural Engineering Conference Modern Agricultural Machinery New Technology Application Seminar, (2010), pp.474-479.

[3] 2012 Heilongjiang Agricultural Machinery Market Terminal Investigation Report, High-end Agricultural Equipment, (2013), Vol.3, pp.47-54.

[4] Wang Chuan, Design and research of agricultural machinery equipment information management system, Anhui Agricultural University, M.S. Thesis, (2009)

[5] Ellerbe Somers Gregg, Jonathan Colton, Md Abdul Matin, and Timothy J. Krupnik, Efficient and participatory design of scale-appropriate agricultural machinery workshops in developing countries: a case study in Bangladesh, Development Engineering, (2019), Vol.5, Article No.100046, DOI: 10.1016/j.deveng.2019.100046 